\title{
International histopathology consensus for unilateral primary aldosteronism
}

Tracy Ann Williams $\mathrm{PhD}^{1,2 *}$, Celso E. Gomez-Sanchez $\mathrm{MD}^{3}$, William E. Rainey PhD ${ }^{4,5}$, Thomas J. Giordano MD PhD ${ }^{5,6}$, Alfred K. Lam MD PhD ${ }^{7}$, Alison Marker $\mathrm{MD}^{8}$, Ozgur Mete $\mathrm{MD}^{9,10}$, Yuto Yamazaki MD PhD ${ }^{11}$, Maria Claudia Nogueira Zerbini MD PhD ${ }^{12}$, Felix Beuschlein MD ${ }^{1,13}$, Fumitoshi Satoh MD PhD ${ }^{14}$, Jacopo Burrello MD², Holger Schneider MD ${ }^{1}$, Jacques W.M. Lenders MD, PhD ${ }^{15,16}$, Paolo Mulatero $\mathrm{MD}^{2}$, Isabella Castellano $\mathrm{MD}^{17}$, Thomas Knösel MD ${ }^{18}$, Mauro Papotti MD ${ }^{19}$, Wolfgang Saeger $\mathrm{MD}^{20}$, Hironobu Sasano MD, $\mathrm{PhD}^{11_{*}^{*}}$, Martin Reincke MD ${ }^{1 \#}$

\section{Disclosure summary: The authors have nothing to disclose}

${ }^{1}$ Medizinische Klinik und Poliklinik IV, Klinikum der Universität München, Ludwig-Maximilians-Universität München, Germany (TA Williams, F Beuschlein, H Schneider, M Reincke)

${ }^{2}$ Division of Internal Medicine and Hypertension, Department of Medical Sciences, University of Turin, Turin, Italy (TA Williams, J Burrello, P Mulatero)

${ }^{3}$ Endocrine Division, G.V. (Sonny) Montgomery VA Medical Center, and Department of Pharmacology and Toxicology and Medicine, University of Mississippi Medical Center, Jackson, MS, USA (CE Gomez-Sanchez)

${ }^{4}$ Department of Molecular and Integrative Physiology, University of Michigan, Ann Arbor (WE Rainey)

${ }^{5}$ Division of Metabolism, Endocrine, and Diabetes, Department of Internal Medicine, University of Michigan, Ann Arbor (WE Rainey and TJ Giordano)

${ }^{6}$ Department of Pathology and Rogel Cancer Center, University of Michigan, Ann Arbor (TJ Giordano)

${ }^{7}$ School of Medicine, Griffith University, Gold Coast, QLD, Australia (AK Lam)

${ }^{8}$ Department of Histopathology, Cambridge University NHS Foundation Trust, Cambridge, UK (A Marker)

${ }^{9}$ Department of Pathology, University Health Network, Toronto, ON, Canada (O Mete)

${ }^{10}$ Department of Laboratory Medicine and Pathobiology, University of Toronto, Toronto, ON, Canada (O Mete)

${ }^{11}$ Department of Pathology, Tohoku University Graduate School of Medicine, Sendai, Japan (Y Yamazaki and H Sasano)

${ }^{12}$ Departamento de Patologia, Faculdade de Medicina da Universidade de Sao Paulo, Sao Paulo, Brazil (MCN Zerbini)

(C) Endocrine Society 2020. All rights reserved. For permissions, please e-mail: journals.permissions@oup.com jc.2020-01643 See https://academic.oup.com/endocrinesociety/pages/Author_Guidelines for Accepted Manuscript disclaimer and additional information. 
${ }^{13}$ Klinik für Endokrinologie, Diabetologie und Klinische Ernährung, Universitätsspital Zürich, Zürich, Switzerland (F Beuschlein)

${ }^{14}$ Division of Clinical Hypertension, Endocrinology and Metabolism, Tohoku University Graduate School of Medicine, Sendai, Japan (F Satoh)

${ }^{15}$ Department of Internal Medicine, Radboud University Medical Center, Nijmegen, The Netherlands (JWM Lenders)

${ }^{16}$ Department of Medicine III, University Hospital Carl Gustav Carus, Technical University Dresden, Dresden, Germany (JWM Lenders)

${ }^{17}$ Division of Pathology, Department of Medical Sciences, University of Turin, Turin, Italy (I Castellano)

${ }^{18}$ Institute of Pathology, Ludwig-Maximilians-Universität München, Germany (T Knösel)

${ }^{19}$ Department of Oncology, University of Turin, Turin, Italy (M Papotti)

${ }^{20}$ Institute of Pathology, Universität Hamburg, Germany (W Saeger)

\section{\# Equal contribution \\ *Co-corresponding}

Tracy Ann Williams PhD, Medizinische Klinik und Poliklinik IV, Klinikum der Universität München, LMU München, Ziemssenstr. 1, D-80336 München, Germany. Tel: +49 894400 52941; Fax: +49 894400 54428; Email: Tracy.Williams@med.uni-muenchen.de

Hironobu Sasano MD, PhD, Department of Pathology, Tohoku University School of Medicine, 2-1 Seiryou-machi, Aoba-Ku, Sendai, Japan 980-8575. Tel : +81-22-717-7450; Fax: +81-22-2735976; Email: hsasano@patholo2.med.tohoku.ac.jp 


\section{Abstract:}

Objective: Develop a consensus for the nomenclature and definition of adrenal histopathologic features in unilateral primary aldosteronism (PA)

Context: Unilateral PA is the most common surgically-treated form of hypertension.

Morphologic examination combined with CYP11B2 (aldosterone synthase) immunostaining reveals diverse histopathologic features of lesions in the resected adrenals.

Patients and Methods: Surgically removed adrenals $(n=37)$ from 90 patients operated from 2015 to 2018 in Munich, Germany, were selected to represent the broad histologic spectrum of unilateral PA. Five pathologists (Group 1 from Germany, Italy and Japan) evaluated the histopathology of haematoxylin-eosin and CYP11B2 immunostained sections and a consensus was established to define the identifiable features. The consensus was subsequently used by six additional pathologists (Group 2 from Australia, Brazil, Canada, Japan, UK, USA) for the assessment of all adrenals with disagreement for histopathologic diagnoses among group 1 pathologists. 
Results: Consensus was achieved to define histopathologic features associated with PA. Use of CYP11B2 immunostaining resulted in a change of the original haematoxylin-eosin morphologydriven diagnosis in 5 (14\%) of 37 cases. Using the consensus criteria, group 2 pathologists agreed for the evaluation of 11 of the 12 cases of disagreement among group 1 pathologists. Conclusion: The HISTALDO (histopathology of primary aldosteronism) consensus is useful to standardize nomenclature and achieve consistency among pathologists for the histopathologic diagnosis of unilateral PA. CYP11B2 immunohistochemistry should be incorporated into the routine clinical diagnostic workup to localize the likely source of aldosterone production.

Key words: Primary aldosteronism, diagnostic histopathology, CYP11B2, adrenal gland, immunohistochemistry 


\section{Introduction:}

Primary aldosteronism (PA) is characterized by hypertension and aldosterone overproduction relative to a suppressed renin-angiotensin system. The main causes of PA are a unilateral aldosterone-producing adenoma (APA) or bilateral adrenal cortical hyperplasia. Other causes could include unilateral adrenal cortical hyperplasia and very rarely, an aldosterone-producing adrenocortical carcinoma (1). Unilateral forms of PA are mainly treated by laparoscopic adrenalectomy of the overactive gland in contrast to pharmacotherapy with mineralocorticoid receptor antagonists for bilateral forms (2).

Aldosterone is produced in the zona glomerulosa (ZG) of the adrenal cortex by common enzymes of all three adrenocortical layers and a specific enzyme of the ZG called aldosterone synthase (CYP11B2, cytochrome P450 family 11, subfamily B, member 2). CYP11B2 displays a high level (93\%) of amino acid sequence similarity to $11 \beta$-hydroxylase (CYP11B1, cytochrome P450 family 11 , subfamily B, member 1 ) which produces cortisol from 11-deoxycortisol located only in the zona fasciculata (3). Haematoxylin-eosin (HE) staining differentiates the relatively small and compact appearance of ZG cells from the larger, lipid-rich and clear cell features of the zona fasciculata (4). Adrenocortical tumours of patients with PA are composed of clear (lipid rich) or compact (eosinophilic) cells or a mixture of both (4-6). The variation in cytomorphology is associated with somatic aldosterone-driver mutations which are prevalent in these tumours (7-9).

The generation and availability of specific monoclonal antibodies to CYP11B2 and CYP11B1 represented a major advance in understanding the pathophysiology of PA (10). Visualization of 
the localization of CYP11B2 in resected adrenals identifies the potential source of aldosterone excess. CYP11B2 immunohistochemistry (IHC) is widely used in scientific studies to examine the functional morphology of normal and pathological adrenals (11-14) and occasionally in surgical pathology for interpretation of the adrenal histology of the resected gland (15). These studies demonstrated the highly variable histopathologic features of surgically resected adrenals from patients with unilateral PA. The classical solitary APA (with homogeneous or heterogeneous CYP11B2 immunoreactivity) is often associated with a hyperplastic ZG negative for CYP11B2immunoreactivity in the same adrenal. Because an atrophic ZG would be expected in the context of a suppressed renin-angiotensin system, this is referred to as paradoxical hyperplasia (16). Other features have been described including multiple macro or micronodules with CYP11B2 positive immunostaining or diffuse hyperplasia (17). Much focus has centered on relatively small micronodules of cells beneath the adrenal capsule with marked CYP11B2 immunoreactivity, usually referred to as aldosterone-producing cell clusters but also as micronodular hyperplasia, foci, or cell nests. These features are present in normal adrenals as well as in the same adrenal as an APA or in adrenals without an adenoma from patients operated for both unilateral and bilateral PA $(8,13,14,18-21)$.

The objectives of this study were to establish consensus among pathologists toward the standardization of nomenclature and definition of adrenal histopathologic features detected in specimens from unilateral PA. We hypothesized that using a uniform terminology and defined criteria should improve consistency and reproducibility of the histopathologic diagnosis of unilateral PA and help classify the likely pathophysiologic source of aldosterone excess. 


\section{Methods:}

The study comprised 3 phases: (I) assessment of the utility of CYP11B2 IHC by a first group of pathologists (from Germany, Italy, and Japan); (II) identification of histopathologic features requiring uniform nomenclature and definition, and consensus criteria building for recommendations; (III) validation of recommendations by histologic consensus-based evaluation of a subset of adrenals by a second group of pathologists with a diverse geographical distribution (Australia, Brazil, Canada, Japan, UK, and USA) (Figure 1).

\section{Patient samples}

The study included 37 patients diagnosed with unilateral PA and treated at Klinikum der Universität München, LMU München, Germany in accordance with the Endocrine Society Guideline (2). Unilateral forms of PA were identified by adrenal venous sampling and treated by total laparoscopic adrenalectomy according to criteria used in the Munich centre (22).

Catheterization of the right adrenal vein was unsuccessful in 3 cases. These 3 patients all presented with a florid phenotype including hypokalaemia. Two cases displayed a contralateral ratio less than 0.8 (where contralateral ratio is defined as the aldosterone/cortisol concentration ratio of the non-dominant adrenal vein divided by the aldosterone/cortisol concentration ratio of a peripheral vein) which may indicate that the source of aldosterone production was the adrenal contralateral to the cannulated gland. In one case, this was also consistent with computer tomography findings of an adrenocortical nodule in the left gland and a normal right gland. The third patient was 42 years-old, with hypertension since age 23 and had a unilateral left nodule at computed tomography imaging. In all 3 cases, the post-surgical 
evaluation demonstrated a complete biochemical remission indicating that the localisation of unilateral disease was correct.

The 37 adrenal specimens were selected from 90 consecutively adrenalectomized patients for unilateral PA over a four-year period from 2015 to 2018. Surgical handling and processing of the resected adrenals was according to standard pathology procedures (23). Whole adrenals were removed by laparoscopic adrenalectomy. All resected adrenals were prospectively sectioned and stained for HE and CYP11B2 IHC for multiple blocks from the same adrenal according to routine practice in the Munich centre. Sample inclusion criteria was based on HE staining and CYP11B2 IHC of the paraffin-embedded adrenals to encompass the entire adrenal pathology spectrum in this cohort over the four-year period and was enriched for unusual cases which pathologists may encounter in this disease (assessed by T Knösel, CE Gomez-Sanchez, and TA Williams).

Written informed consent for the scientific study of adrenal specimens (including genotype analysis and histopathology) and use of patient data was approved by the local ethics committee.

\section{Evaluation of the utility of CYP11B2 immunohistochemistry}

Whole slide images were created by scanning the complete histologic slide to produce highresolution digital files of the HE and CYP11B2 immunostained sections. Images were navigated using Aperio ImageScope software. The utility of CYP11B2 IHC was evaluated by comparing two rounds of adrenal assessment: with HE staining alone (phase I, round A) and with the additional 
examination of CYP11B2 IHC (phase I, round B) (Figure 1). The pathologists were blinded to the CYP11B2 IHC during the first-round evaluation of HE slides.

In round $A$, whole slide images stained with $\mathrm{HE}$ were independently evaluated by five pathologists (Group 1 pathologists: H. Sasano [Sendai, Japan], W. Saeger [Hamburg, Germany], M. Papotti and I. Castellano [Torino, Italy], and T. Knösel [Munich, Germany]) and examined for histopathologic findings of "classical" (solitary well circumscribed APA) or "non-classical" (absence of an APA) unilateral PA. Evaluations were returned to the core group (CE GomezSanchez, WE Rainey, M Reincke, TA Williams) before round B in which the same 37 adrenals were examined by group 1 pathologists based on both HE staining and CYP11B2 IHC.

Histopathologic features of these adrenal specimens were identified for the development of consensus recommendations from the histopathologic assessments of group 1 pathologists by the core group. The histologic findings referred to as classical or non-classical unilateral PA based on HE alone or with HE and CYP11B2 immunostaining were then compared to assess the utility of CYP11B2 IHC.

\section{Assessment of agreement level between group 1 pathologists}

Agreement between pathologists for the assessment of each adrenal was defined when four or more group 1 pathologists $(n=6)$ reached the same histopathologic diagnosis of classical unilateral PA or non-classical. Cases with disagreement were used for the validation phase by group 2 pathologists. 


\section{Consensus building for nomenclature and definition of features}

To achieve definitions for specific histopathologic features in the adrenals with PA, group 1 pathologists responded to an open questionnaire requesting criteria for the differentiation between CYP11B2 positive lesions identified from their assessments. Putative definitions were derived from responses and feedback from expert clinicians in PA and adrenal experts was requested (CE Gomez-Sanchez, JWM Lenders, P Mulatero, WE Rainey). A dichotomous questionnaire to assess agreement or disagreement with the proposed criteria was sent to group 1 pathologists with comments from clinicians and adrenal experts.

A subsequent face-to-face meeting for the discussion of all nomenclature and histopathologic criteria of features was held in Munich, Germany (Medizinische Klinik und Poliklinik IV, Klinikum der Universität München, LMU München) on $13^{\text {th }}$ October 2019. The core group (CE GomezSanchez, WE Rainey, M Reincke, TA Williams), group 1 pathologists (I Castellano, T Knösel, M Papotti, W Saeger, H Sasano), clinical advisors (JWM Lenders, P Mulatero) were all in attendance.

\section{Validation of consensus}

The consensus criteria were subsequently validated by a group of six additional pathologists (group 2 pathologists) selected to cover a wide geographical distribution over five continents (TJ Giordano [Ann Arbor, USA], AK Lam [Gold Coast, Australia], A Marker [Cambridge, UK], O Mete [Toronto, Canada], Y. Yamazaki [Sendai, Japan], MC Nogueira Zerbini [Sao Paolo, Brazil]). Group 2 pathologists were asked to evaluate whole slide images of matched HE and CYP11B2 immunostained slides from 18 of the original set of 37 adrenals evaluated by group 1 
pathologists. The 18 adrenals comprised all 12 adrenals for which group 1 pathologists showed disagreement and 6 adrenals for which the Group 1 pathologists had reached agreement ( 3 cases of agreement for histopathologic findings of "classical" unilateral PA; 3 cases of agreement for "non-classical” unilateral PA).

The pathologists involved in this study had a special interest in adrenal pathology, but for some of them, the adrenal was not their primary research interest. All pathologists were blinded to the others' diagnoses.

\section{Statistical analyses}

Unadjusted analyses were performed using IBM SPSS Statistics version 22.0. Quantitative normally distributed variables are shown as means with SDs; quantitative non-normally distributed variables are given as medians and IQRs. Categorical variables are shown as absolute numbers and percentages. A student t-test was used to compare quantitative normally distributed variables. A Mann-Whitney $U$ test was applied for the analysis of group differences of quantitative non-normally distributed variables and a Chi-square or Fisher's exact tests for categorical variables. The absolute number and proportion of patients in each postsurgical outcome category (complete, partial and absent clinical and biochemical success at 612 months after surgery) were assessed by the PASO criteria (24). Patients classified with absent biochemical success after surgery all failed to suppress aldosterone production below $277 \mathrm{pmol} / \mathrm{L}(10 \mathrm{ng} / \mathrm{dL})$ with a post-surgical saline infusion confirmatory test (2). 


\section{Results:}

Supplemental tables and figures can be downloaded directly at:

https://github.com/MedIVLMUMunich/HISTALDOconsensus (25).

Phase I - Assessment of adrenalectomy specimens and identification of histopathologic features requiring uniform nomenclature and definition

With HE-based assessment alone (Phase I, Round A), group 1 pathologists agreed with the histopathologic diagnosis in 21 (56.8\%) of 37 cases, but, disagreed in the remaining 16 (43.2\%) cases. The 21 cases consisted of 18 diagnoses of classical histopathologic findings of unilateral $\mathrm{PA}$, and the three remaining cases were non-classical histopathologic findings. With the additional examination of CYP11B2 IHC (Phase I, Round B), disagreement between group 1 pathologists was reduced from $16(43.2 \%)$ to $12(32.4 \%)$ of 37 cases. The 25 cases with mutual agreement (67.6\%) comprised 16 adrenals with histopathologic findings of classical unilateral disease, and nine non-classical cases (Figure 2).

Comparison of adrenal scoring based on HE staining alone (round A) with assessment including CYP11B2 IHC (round B, group 1 pathologists) demonstrated that the visualization of CYP11B2 
(aldosterone synthase) resulted in three or more of the five pathologists changing their original histopathologic diagnosis for five cases (14\%). These cases comprised adrenals \#10, \#11, \#14, $\# 19$ and \#20 (25, Figures S1 and S2).

Histopathologic features derived from the evaluations included the following terminologies: (i) APA, (ii) non-functioning adenoma, (iii) CYP11B2 positive nodule, (iv) aldosterone-producing cell clusters, (v) CYP11B2 positive micronodule, (vi) micronodular hyperplasia and (vii) diffuse hyperplasia.

\section{Phase II - consensus building for recommendations}

After two rounds of questionnaires and the face-to-face meeting, unanimous consensus was reached for recommendations for nomenclature and for the distinction of specific lesions (Table 1). This resulted in the criteria below for the histopathology of unilateral PA.

\section{Aldosterone-producing adenoma:}

An APA is a solitary neoplasm of at least $10 \mathrm{~mm}$ diameter composed of clear cells, compact eosinophilic cells, or a mixture of both (26). An APA and a nonfunctioning adenoma are morphologically similar and cannot be differentiated by HE staining alone. Differentiation is based on clinical information including functional assessment of aldosterone overproduction by adrenal venous sampling and histologic findings of CYP11B2 positive immunostaining in tumour cells using a validated CYP11B2 antibody (10) (Figure 3). Terminology such as "glomerulosalike" and "fasciculata-like" should not be used for the description of small compact or lipid-rich cells of an APA in favour of the correct pathologic description of compact eosinophilic or clear cells in surgical pathology reports. Macroscopic criteria relating to the colour of the cut surface 
of the adrenal specimen and microscopic criteria related to the presence of spironolactone bodies in tumour cells are unreliable and should not be considered in the diagnosis of APA.

\section{Aldosterone-producing nodule:}

An aldosterone-producing nodule is a CYP11B2 positive lesion of less than $10 \mathrm{~mm}$ diameter composed of clear cells, compact eosinophilic cells, or a mixture of both. An aldosteroneproducing nodule and an APA display similar morphology but HE staining can help distinguish the smaller size of a nodule from an adenoma if the lesion has been sectioned appropriately at its greatest diameter. Aldosterone-producing nodules often show polarity of CYP11B2 immunostaining with decreasing intensity of immunoreactivity from the outer to the inner part of the lesion (Figure 3). This contrasts with the CYP11B2 immunoreactivity in most APAs in which a gradient of CYP11B2-positive staining is absent and replaced by either a homogeneous or a diffusely heterogeneous pattern of CYP11B2 immunoreactivity throughout the lesion.

\section{Aldosterone-producing micronodule:}

An aldosterone-producing micronodule is a CYP11B2 positive lesion measuring less than $10 \mathrm{~mm}$ (in the greatest dimension), located in the outer margin of the subcapsular ZG (Figure 3). An aldosterone-producing micronodule cannot be distinguished by HE from the surrounding ZG. In contrast, an aldosterone-producing nodule is morphologically visible with HE staining and can be located elsewhere within the adrenal cortex. Therefore, both CYP11B2 IHC and HE staining are needed to distinguish these two lesions. Aldosterone-producing micronodules also often show polarity of CYP11B2 immunostaining with decreasing intensity of immunoreactivity from the outer to the inner part of the lesion. There is no evidence that an aldosterone-producing 
micronodule can be distinguished from the feature described in the scientific literature as an aldosterone-producing cell cluster. The term "cell cluster" is an unclear term which does not describe the histology and therefore, "aldosterone-producing cell cluster" should be replaced by the recommended term of "aldosterone-producing micronodule".

\section{Multiple aldosterone-producing nodules or micronodules:}

Multiple aldosterone-producing nodules or micronodules are characterized by the separation of aldosterone-producing nodules or micronodules by histologically normal non-hyperplastic ZG (Figure 3). The terminology "nodular hyperplasia" or "micronodular hyperplasia" should be replaced by the recommended terms of "multiple aldosterone-producing nodules" or "multiple aldosterone-producing micronodules" which describe the histologic features of these lesions more precisely. When aldosterone-producing nodules and aldosterone-producing micronodules are found concurrently, the histologic findings should be described as multiple aldosteroneproducing nodules and micronodules.

\section{Aldosterone-producing diffuse hyperplasia:}

Aldosterone-producing diffuse hyperplasia is distinguished from multiple aldosteroneproducing micronodules by both cytomorphology and CYP11B2 IHC. Aldosterone-producing diffuse hyperplasia is the occurrence of a broad and uninterrupted strip of hyperplastic ZG cells with CYP11B2 positive immunostaining in more than $50 \%$ of cells (Figure 3 ). The term aldosterone-producing diffuse hyperplasia is applied irrespective of the presence of aldosterone-producing nodules in the same adrenal gland. 


\section{Importance of tissue handling and sectioning:}

During the face-to-face meeting, tissue handling, and sectioning of surgically removed adrenal specimens were also discussed.

Pre-analytical conditions affect the results of CYP11B2 IHC and recommendations for tissue preparation and fixation are the same as described in detail elsewhere (23). Pathology reporting should follow standard procedures. Histologic diagnosis is more challenging by adrenal resection in multiple pieces. Surgical removal of the entire adrenal specimen in a single piece including the tumour nodule with the attached adrenal cortex with minimal damage is recommended for improved morphologic assessment. Equatorial and vertical sectioning of adrenal tissue is recommended because tangential sections may distort the architecture of lesions. Sectioning should be performed at the greatest lesion diameter to avoid misclassification of an adenoma (at least $10 \mathrm{~mm}$ diameter) as a nodule (less than $10 \mathrm{~mm}$ diameter).

The whole surgically resected specimen should be submitted for histopathologic examination to allow the management of conventional cases with different protocols versus complex cases. Thus, solitary APAs with classical pathological features can be processed with a limited number of sections because there is a low risk of missing relevant histological data in the deeper levels of the paraffin block. Conversely, for more complex specimens, especially those with apparently negative CYP11B2 immunostaining, analyzing the entire specimen on multiple sectioning maximizes the possibility of visualizing the culprit lesion with CYP11B2 IHC. 


\section{Phase III - validation of consensus}

The consensus criteria and recommendations (Panel 1) were provided to group 2 pathologists for the evaluation of whole slide images generated from HE stained and CYP11B2 immunostaining of all 12 adrenals with disagreement for histopathologic assessment between group 1 pathologists as well as six additional adrenals with agreement (Figure 2). Agreement between pathologists was maintained for these six cases by the group 2 pathologists. In the assessment of the 12 adrenals with disagreement between group 1 pathologists, using the consensus criteria, at least five of the six group 2 pathologists agreed for the histopathologic diagnosis of nine adrenals and four pathologists agreed for the assessment of two of the remaining three adrenals. Disagreement was maintained for the classification of adrenal \#18 as classical versus non-classical unilateral PA (25, Figure S3). The individual pathologists' assessments are shown in Table S2 (25). This adrenal was resected from a patient with complete biochemical success and partial clinical success after surgery.

\section{Pathology findings of resected adrenals}

Among 24 adrenals categorized as classical histopathology of unilateral PA, 21 had an APA and the remaining three had an aldosterone-producing cortical nodule (25, Figure S1). The nontumorous adrenal cortex adjacent to the APA frequently displayed paradoxical ZG hyperplasia with negative CYP11B2 immunostaining, aldosterone-producing micronodules or nodules or aldosterone-producing diffuse hyperplasia (25, Figure S1).

Among 12 adrenals with features of non-classical unilateral PA, three had aldosteroneproducing diffuse hyperplasia (adrenals \#4, \#9, and \#29), two showed a nonfunctioning cortical 
adenoma with aldosterone-producing diffuse hyperplasia in the adjacent cortex (\#8, and \#19), and the remaining seven cases had multiple aldosterone-producing nodules (\#5, \#6, \#21, and \#31), aldosterone-producing micronodules (\#30) or a mixed phenotype of aldosteroneproducing nodules and aldosterone-producing micronodules (\#7 and \#33) (25, Table S1, Figure S2).

\section{Clinical parameters stratified by histopathologic diagnosis}

Unadjusted clinical data at baseline and follow-up are shown for all patients with interpathologist agreement with respect to histopathologic findings (Table 2). The histopathologic diagnosis of classical unilateral PA was identified in 24 of the 36 cases and a non-classical histopathology was assigned to 12 cases (Figure 2). There was no agreement among pathologists for the histopathology of the remaining case (25, Figure S3). Therefore, from pathology assessments, this adrenal could not be categorized as either classical or non-classical unilateral PA and was excluded from the descriptive statistical analysis (Table 2).

Patients with histopathologic findings of non-classical unilateral PA displayed a longer known duration of hypertension compared with the classical group (114 months [48 to 176] versus 11 months [3 to 100], $p=0.010$ ) and a smaller nodule size at pathology ( $6 \mathrm{~mm}$ [3 to 10] versus 12 $\mathrm{mm}$ [ 8 to 20], $\mathrm{p}=0.019$ ). The lateralization index (a ratio indicating the level of asymmetry of aldosterone production from the adrenal glands) was also lower in the non-classical compared with the classical group (5.8 [4.4 to 11.5 ] versus 13.9 [6.7 to 36.2 ], $p=0.048$ ). The serum potassium ion concentration at baseline was relatively higher in the non-classical compared with the classical group ( $3.4 \mathrm{mmol} / \mathrm{L} \pm 0.62$ versus $3.0 \pm 0.43$ ), but lower at 6 - 12 months after 
surgery $(3.9 \mathrm{mmol} / \mathrm{L} \pm 0.42$ versus $4.4 \mathrm{mmol} / \mathrm{L} \pm 0.41, p=0.006)$. During the post-surgical followup, the non-classical group also displayed a higher aldosterone-to-renin ratio (used as a screening test for PA) than the classical group (48 [28 to 115] versus 21 [6 to 32], $p=0.006$ ).

Post-surgical follow-up data was available for 34 of the 36 patients with agreement among pathologists for the histopathologic assessment. The two patients with missing follow-up data were included because the adrenals were considered of interest for histopathologic findings of a principal CYP11B2 positive lesion with additional CYP11B2 positive lesions of different forms and dimensions in the adjacent cortex.

The assessment of post-surgical outcomes using the PASO criteria (24) demonstrated no significant differences in clinical outcomes between the two groups $(p=0.286)$ but biochemical outcomes were different ( $p=0.009$ for group difference). Complete biochemical success (biochemical remission) was achieved in $81.9 \%$ (18 of 22) of patients with classical histopathologic findings of unilateral PA compared with $33.3 \%$ (4 of 12) of patients in the nonclassical group ( $(p=0.008)$ (Table 2). A higher proportion of patients with a non-classical histopathology of unilateral PA displayed absent biochemical success after surgery $(41.7 \%, 5$ of 12 patients) than patients in the classical group $(4.5 \%, 1$ of 22 patients, $p=0.014)$ (Table 2 ). 


\section{Discussion:}

This study developed consensus recommendations for the nomenclature and definitions of the histopathologic features associated with unilateral PA. The consensus was built and validated with the participation of an international group of expert pathologists from five continents (Asia, Australia, Europe, North America, and South America) and contributed to an improved consistency among pathologists for the histopathologic diagnosis of this disease.

The pathologic examination of the adrenalectomy specimens from patients with unilateral PA identifies the morphologic changes associated with the disorder. IHC for CYP11B2 (aldosterone synthase), which catalyzes the terminal, and rate limiting steps of aldosterone biosynthesis, is not widely used in clinical pathology but helps visualize the most likely site of aldosterone production $(10,11,27)$. For instance, in an early study in which a patient's adrenal was subjected to CYP11B2 IHC staining, aldosterone-producing micronodules were the presumed source of aldosterone overproduction rather than the primary cortical macronodule which was negative for CYP11B2 (16). This finding is clinically relevant because an adrenal-sparing surgical approach, guided by computed tomography imaging data rather than total adrenalectomy, may not necessarily remove the culprit lesion.

Studies with CYP11B2 immunostaining on normal and pathological adrenal glands have also demonstrated the frequent presence of aldosterone-producing micronodules $(18,19)$. These micronodules are widely referred to as aldosterone-producing cell clusters but the term "cell cluster" is ambiguous and not used in pathology and therefore should be replaced with aldosterone-producing micronodule. Aldosterone-producing micronodules have also been 
reported in adrenals without an APA from patients with preoperative unilateral PA (13), in a small series of adrenals from patients with presurgical bilateral PA (21) and in normal adrenals from kidney donors (20). The aldosterone-producing micronodules in the adrenals from these different groups of patients and individuals appear to differ by the prevalence of mutations in the genes implicated in constitutive aldosterone production (primarily in CACNA1D encoding the calcium channel Cav1.3), by their size or relative number of aldosterone-producing micronodules per adrenal $(20,21)$. In addition, a subset of APMs display convergent in situ metabolic profiles to APAs (28) and may represent the progression of APMs to APAs (29).

The current study showed that the evaluation of CYP11B2 immunostaining in addition to routine morphologic assessment based on HE staining resulted in a change of the original histopathologic interpretation in $14 \%$ of patients. In addition, this approach also highlighted several cases of a nonfunctional adrenocortical adenoma in association with an aldosteroneproducing nodule, multiple aldosterone-producing micronodules or aldosterone-producing diffuse hyperplasia. These findings above underscore the utility of CYP11B2 IHC in the diagnostic workup of unilateral PA.

This series also demonstrated an association of histopathology with post-surgical biochemical outcomes. Around $40 \%$ of cases with non-classical histopathologic findings of unilateral PA (absence of an APA) were associated with post-surgical absent biochemical success (indicating persistent aldosteronism) compared with under $5 \%$ with a classical histopathology of a solitary APA. Because biochemical outcomes indicate if the presurgical diagnosis of unilateral disease was accurate, histopathology may compliment biochemical follow-up to provide reassurance 
that the clinical diagnosis was correct or highlight patients requiring close post-surgical followup $(14,30)$

This is also clinically relevant because PA is associated with a high incidence of cardiovascular, metabolic and renal complications (31) and incomplete treatment is associated with an increased rate of adverse events (32).

For the first time since the historical description of adrenocortical pathology in 1985 by Neville and O'Hare (4), this study provides consensus recommendations to define the various pathologic findings in adrenals from patients with unilateral PA which include immunohistochemical evaluation with a CYP11B2 monoclonal antibody available since 2014 (10).

The strengths of our study include the participation of 11 pathologists from five continents to evaluate the adrenals using stringent criteria to assess agreement for histopathologic diagnoses. In addition, a sample set of adrenal specimens was assessed covering the broad spectrum of adrenal histopathology with a wide variety of features. A further strength is the independent assessment of morphology from CYP11B2 immunostaining which allowed us to assess the utility of functional immunostaining.

The main limitation of the study in the clinical setting is the inability to provide consensus criteria for the histopathology of adrenals from patients operated for bilateral PA because these patients are rarely operated and tissue samples are usually unavailable. 
In conclusion, the HISTALDO consensus provides recommendations and criteria for the histopathologic diagnosis of unilateral PA. We identified the main histologic features associated with this disease and demonstrated that using a standardized set of criteria improves diagnostic agreement between pathologists. Our findings demonstrate the relevance of histopathology with morphologic and IHC evaluation in the clinical management of unilateral PA. Histopathologic examination of adrenalectomy specimens with CYP11B2 IHC is therefore recommended for all patients operated for unilateral PA. 


\section{Acknowledgements:}

The technical assistance of Isabella-Sabrina Kinker and the support of the clinical primary aldosteronism team in Munich are gratefully acknowledged.

\section{Glossary:}

APA aldosterone-producing adenoma

APACC aldosterone-producing adrenocortical carcinoma

APDH aldosterone-producing diffuse hyperplasia

APM aldosterone-producing micronodule

APN aldosterone-producing nodule

ARR aldosterone-to-renin ratio

BMI body mass index

BP blood pressure

DDD defined daily dose

DRC direct renin concentration

HE haematoxylin-eosin

IHC immunohistochemistry

HTN hypertension

MAPM multiple aldosterone-producing micronodules

MAPN multiple aldosterone-producing nodules

PA primary aldosteronism

ZG zona glomerulosa 


\section{Financial Support:}

This work was supported by the European Research Council (ERC) under the European Union's Horizon 2020 research and innovation programme (grant agreement No [694913] to M Reincke) and by the Deutsche Forschungsgemeinschaft (DFG, German Research Foundation) project number: 314061271-TRR 205 to F Beuschlein, JWM Lenders, M Reincke and TA Williams and the Clinician Scientist PRogram In Vascular MEdicine (PRIME) project number MA 2186/141 to H Schneider. This work was also supported by the Else Kröner-Fresenius Stiftung in support of the German Conn Registry-Else-Kröner Hyperaldosteronism Registry (2015_A171, and 2019_A104 to M Reincke) and by the Clinical Research Priority Program of the University of Zurich for the CRPP Hypertension Research Network (HYRENE) to F Beuschlein. CE GomezSanchez is supported by National Heart, Lung and Blood Institute grant R01 HL144847, and the National Institute of General Medical Sciences grant U54 GM115428 and WE Rainey is supported by National Institute of Diabetes and Digestive and Kidney Diseases grant R01 DK106618. F Satoh is supported by JSPS KAKENHI (Japan Society for the Promotion of Science, Grants-in-Aid for Scientific Research) grant number JP18K08500 and Health Labour Sciences Research grant number H29-Nanji-Ippan-046. 


\section{References:}

(1) Mulatero P, Monticone S, Rainey WE, Veglio F, Williams TA. Role of KCNJ5 in familial and sporadic primary aldosteronism. Nat Rev Endocrinol. 2013;9(2):104-112.

(2) Funder JW, Carey RM, Mantero F, Murad MH, Reincke M, Shibata H, Stowasser M, Young WF Jr. The management of primary aldosteronism: case detection, diagnosis, and treatment: an Endocrine Society clinical practice guideline. J Clin Endocrinol Metab. 2016;101(5):1889-1916.

(3) Stowasser M, Gordon RD. Primary aldosteronism: changing definitions and new concepts of physiology and pathophysiology both inside and outside the kidney. Physiol Rev. 2016;96(4):1327-1384.

(4) Neville AM, O'Hare MJ. Histopathology of the human adrenal cortex. Clin Endocrinol Metab. 1985;14(4):791-820.

(5) Gordon RD, Klemm SA, Tunny TJ, Stowasser M. Primary aldosteronism: hypertension with a genetic basis. Lancet. 1992;340(8812):159-161.

(6) Gordon RD, Stowasser M, Klemm SA, Tunny TJ. Primary aldosteronism-some genetic, morphological, and biochemical aspects of subtypes. Steroids. 1995;60(1):35-41.

(7) Azizan EA, Lam BY, Newhouse SJ, Zhou J, Kuc RE, Clarke J, Happerfield L, Marker A, Hoffman GJ, Brown MJ. Microarray, qPCR, and KCNJ5 sequencing of aldosterone-producing adenomas reveal differences in genotype and phenotype between zona glomerulosa- and zona fasciculatalike tumors. J Clin Endocrinol Metab. 2012;97(5):E819-E829.

(8) Monticone S, Castellano I, Versace K, Lucatello B, Veglio F, Gomez-Sanchez CE, Williams TA, Mulatero P. Immunohistochemical, genetic and clinical characterization of sporadic aldosterone-producing adenomas. Mol Cell Endocrinol. 2015;411:146-154. 
(9) Ono Y, Yamazaki Y, Omata K, Else T, Tomlins SA, Rhayem Y, Williams TA, Reincke M, Carling T, Monticone S, Mulatero P, Beuschlein F, Ito S, Satoh F, Rainey WE, Sasano H. Histological characterization of aldosterone-producing adrenocortical adenomas with different somatic mutations. J Clin Endocrinol Metab. 2020;105(3):e282-e289.

(10) Gomez-Sanchez CE, Qi X, Velarde-Miranda C, Plonczynski MW, Parker CR, Rainey W, Satoh F, Maekawa T, Nakamura Y, Sasano H, Gomez-Sanchez EP. Development of monoclonal antibodies against human CYP11B1 and CYP11B2. Mol Cell Endocrinol. 2014;383(1-2):111-117. (11) Nakamura Y, Maekawa T, Felizola SJ, Satoh F, Qi X, Velarde-Miranda C, Plonczynski MW, Ise K, Kikuchi K, Rainey WE, Gomez-Sanchez EP, Gomez-Sanchez CE, Sasano H. Adrenal CYP11B1/2 expression in primary aldosteronism: immunohistochemical analysis using novel monoclonal antibodies. Mol Cell Endocrinol. 2014;392(1-2):73-79.

(12) Fernandes-Rosa FL, Giscos-Douriez I, Amar L, Gomez-Sanchez CE, Meatchi T, Boulkroun S, Zennaro MC. Different somatic mutations in multinodular adrenals with aldosterone-producing adenoma. Hypertension. 2015;66(5):1014-1022.

(13) Yamazaki Y, Nakamura Y, Omata K, Ise K, Tezuka Y, Ono Y, Morimoto R, Nozawa Y, GomezSanchez CE, Tomlins SA, Rainey WE, Ito S, Satoh F, Sasano H. Histopathological classification of cross-sectional image-negative hyperaldosteronism. J Clin Endocrinol Metab. 2017;102(4):11821192.

(14) Meyer LS, Wang X, Sušnik E, Burrello J, Burrello A, Castellano I, Eisenhofer G, Fallo F, Kline GA, Knösel T, Kocjan T, Lenders JWM, Mulatero P, Naruse M, Nishikawa T, Peitzsch M, Rump LC, Beuschlein F, Hahner S, Gomez-Sanchez CE, Reincke M, Williams TA. Immunohistopathology 
and steroid profiles associated with biochemical outcomes after adrenalectomy for unilateral primary aldosteronism. Hypertension. 2018;72(3):650-657.

(15) Volpe C, Höög A, Ogishima T, Mukai K, Lu M, Thorén M, Hamberger B.

Immunohistochemistry improves histopathologic diagnosis in primary aldosteronism. J Clin Pathol. 2013;66(4):351-354.

(16) Ito A, Yamazaki Y, Sasano H, Matsubara D, Fukushima N, Tamba M, Tabata K, Ashizawa K, Takei A, Koizumi M, Sakuma Y, Sata N, Oshiro H. A case of primary aldosteronism caused by unilateral multiple adrenocortical micronodules presenting as muscle cramps at rest: The importance of functional histopathology for identifying a culprit lesion. Pathol Int. 2017;67(4):214-221.

(17) Gomez-Sanchez CE, Kuppusamy M, Reincke M, Williams TA. Disordered CYP11B2 expression in primary aldosteronism. Horm Metab Res. 2017;49(12):957-962.

(18) Nishimoto K, Nakagawa K, Li D, Kosaka T, Oya M, Mikami S, Shibata H, Itoh H, Mitani F, Yamazaki T, Ogishima T, Suematsu M, Mukai K. Adrenocortical zonation in humans under normal and pathological conditions. J Clin Endocrinol Metab. 2010;95(5):2296-2305. (19) Boulkroun S, Samson-Couterie B, Dzib JF, Lefebvre H, Louiset E, Amar L, Plouin PF, Lalli E, Jeunemaitre X, Benecke A, Meatchi T, Zennaro MC. Adrenal cortex remodeling and functional zona glomerulosa hyperplasia in primary aldosteronism. Hypertension. 2010;56(5):885-892. (20) Nishimoto K, Tomlins SA, Kuick R, Cani AK, Giordano TJ, Hovelson DH, Liu CJ, Sanjanwala AR, Edwards MA, Gomez-Sanchez CE, Nanba K, Rainey WE. Aldosterone-stimulating somatic gene mutations are common in normal adrenal glands. Proc Natl Acad Sci U S A.

2015;112(33):E4591-E4599. 
(21) Omata K, Satoh F, Morimoto R, Ito S, Yamazaki Y, Nakamura Y, Anand SK, Guo Z, Stowasser M, Sasano H, Tomlins SA, Rainey WE. Cellular and genetic causes of idiopathic hyperaldosteronism.

Hypertension. 2018;72(4):874-880.

(22) Williams TA, Reincke M. MANAGEMENT OF ENDOCRINE DISEASE: Diagnosis and management of primary aldosteronism: the Endocrine Society guideline 2016 revisited. Eur J Endocrinol. 2018;179(1):R19-R29.

(23) Lam A, Chong G, Dahlstrom J, McNicol AM, Gill A, Sullivan L. Adrenal gland tumours structured protocol, 1st edition, 2013 Published on web by Royal College of Pathologists of Australasia. http://www.rcpa.edu.au//Library/Practising-Pathology/Structured-Pathology$\underline{\text { Reporting-of-Cancer/Cancer-Protocols }}$

(24) Williams TA, Lenders JWM, Mulatero P, Burrello J, Rottenkolber M, Adolf C, Satoh F, Amar L, Quinkler M, Deinum J, Beuschlein F, Kitamoto KK, Pham U, Morimoto R, Umakoshi H, Prejbisz A, Kocjan T, Naruse M, Stowasser M, Nishikawa T, Young WF Jr, Gomez-Sanchez CE, Funder JW, Reincke M; Primary Aldosteronism Surgery Outcome (PASO) investigators. Outcomes after adrenalectomy for unilateral primary aldosteronism: an international consensus on outcome measures and analysis of remission rates in an international cohort. Lancet Diabetes Endocrinol. 2017;5(9):689-699.

(25) Williams TA, Gomez-Sanchez CE, Rainey WE, Giordano TJ, Lam AK, Marker A, Mete O, Yamazaki Y, Zerbini MCN, Beuschlein F, Satoh F, Burrello J, Schneider H, Lenders JWM, Mulatero P, Castellano I, Knösel T, Papotti M, Saeger W, Sasano H, Reincke M. Data from: International histopathology consensus for unilateral primary aldosteronism. Deposited 10th June 2020 
https://github.com/MedIVLMUMunich/HISTALDOconsensus/raw/master/Supplemental\%20dat a.pdf

(26) Yamazaki Y, Omata K, Tezuka Y, Ono Y, Morimoto R, Adachi Y, Ise K, Nakamura Y, GomezSanchez CE, Shibahara Y, Kitamoto T, Nishikawa T, Ito S, Satoh F, Sasano H. Tumor cell subtypes based on the intracellular hormonal activity in KCNJ5-mutated aldosterone-producing adenoma.

Hypertension. 2018;72(3):632-640.

(27) Seccia TM, Caroccia B, Gomez-Sanchez EP, Gomez-Sanchez CE, Rossi GP. The biology of normal zona glomerulosa and aldosterone-producing adenoma: pathological implications. Endocr Rev. 2018;39(6):1029-1056.

(28) Sun N, Meyer LS, Feuchtinger A, Kunzke T, Knösel T, Reincke M, Walch A, Williams TA. Mass spectrometry imaging establishes 2 distinct metabolic phenotypes of aldosterone-producing cell clusters in primary aldosteronism. Hypertension. 2020;75(3):634-644.

(29) Nishimoto K, Seki T, Kurihara I, Yokota K, Omura M, Nishikawa T, Shibata H, Kosaka T, Oya M, Suematsu M, Mukai K. Case report: nodule development from subcapsular aldosteroneproducing cell clusters causes hyperaldosteronism. J Clin Endocrinol Metab. 2016;101(1):6-9. (30) Volpe C, Hamberger B, Höög A, Mukai K, Calissendorff J, Wahrenberg H, Zedenius J, Thorén M. Primary aldosteronism: functional histopathology and long-term follow-up after unilateral adrenalectomy. Clin Endocrinol (Oxf). 2015;82(5):639-647.

(31) Monticone S, D'Ascenzo F, Moretti C, Williams TA, Veglio F, Gaita F, Mulatero P. 
Cardiovascular events and target organ damage in primary aldosteronism compared with essential hypertension: a systematic review and meta-analysis. Lancet Diabetes Endocrinol. 2018;6(1):41-50.

(32) Hundemer GL, Curhan GC, Yozamp N, Wang M, Vaidya A. Cardiometabolic outcomes and mortality in medically treated primary aldosteronism: a retrospective cohort study. Lancet Diabetes Endocrinol. 2018;6(1):51-59. 


\section{Figure Legends:}

\section{Figure 1: HISTALDO consensus procedure}

Paraffin-embedded adrenals were selected from HE staining and CYP11B2 immunohistochemistry to cover the wide spectrum of adrenal histopathology observed in surgically-treated patients for unilateral PA (37 of 90 adrenals). Whole slide images of the $37 \mathrm{HE}$-stained adrenals were assessed by 5 pathologists (group 1 pathologists, round A) for histopathology associated with "classical" or "nonclassical" unilateral PA. Group 1 pathologists subsequently assessed the same adrenals based on HE and CYP11B2 (aldosterone synthase) immunostaining (round B) to evaluate the utility of CYP11B2 IHC in the diagnostic pathology of unilateral PA. Diverse features associated with unilateral PA for the consensus building phase were identified from histopathologic findings. Consensus was achieved by two rounds of questionnaires comprising an open questionnaire, a dichotomous questionnaire (agree or disagree) and a face-to-face meeting (see Methods for further details). The consensus criteria were provided to a group of 6 additional pathologists (group 2 pathologists) as a guide for HE and CYP11B2 IHC-based examination of 18 of the 37 adrenals assessed by the group 1 pathologists. The 18 adrenals comprised 6 adrenals for which group 1 pathologists showed agreement ( 3 cases of agreement for classical and 3 cases of agreement for non-classical histology of unilateral PA (25, adrenals \#1 to \#6) and all 12 adrenals for which group 1 pathologists showed a high level of disagreement (25, adrenals \#7 to \#18). 
Figure 2. Agreement between group 1 and group 2 pathologists for the histopathologic diagnosis of unilateral primary aldosteronism.

Agreement was defined as at least 4 of the 5 group 1 pathologists or at least 4 of the 6 group 2 pathologists reaching the same histopathologic diagnosis. Classical, indicates "classical" histopathology associated with unilateral PA of a solitary APA or APN; non-classical, indicates "non-classical" histopathology of MAPM or MAPN (or MAPM and MAPN together) or APDH.

Figure 3. Histopathologic features in surgically removed adrenals from patients with unilateral primary aldosteronism

Paraffin-embedded adrenal sections $(3 \mu \mathrm{m})$ were processed and stained for haematoxylin-eosin (H\&E) and immunostained for CYP11B2 (aldosterone synthase) using the mouse monoclonal anti-human CYP11B2 antibody (clone 17B) (10). APAs are shown with homogeneous (Panel A) and heterogeneous (Panel B) CYP11B2 immunostaining. The CYP11B2 positive immunostaining in tumour cells distinguishes an APA from a nonfunctioning adenoma (Panel C). APNs (aldosterone-producing nodules) are morphologically visible with HE staining (Panel D) whereas APMs (aldosterone-producing micronodules) are morphologically indistinct from adjacent adrenocortical cells (Panel E).

Black scale bar, $2 \mathrm{~mm}$; white scale bar, $200 \mu \mathrm{m}$. 


\section{Table 1. HISTALDO consensus for nomenclature and histopathology of adrenal cortical lesions in unilateral primary aldosteronism.}

\begin{tabular}{|c|c|c|}
\hline Histopathological entity & Abbreviation & Definition \\
\hline $\begin{array}{l}\text { Aldosterone-producing } \\
\text { adrenocortical carcinoma }\end{array}$ & APACC & $\begin{array}{l}\text { Aldosterone-producing malignant neoplasms, including } \\
\text { variants, follow the same pathologic criteria of other } \\
\text { adrenocortical cancers. }\end{array}$ \\
\hline Aldosterone-producing adenoma & APA & $\begin{array}{l}\text { Well circumscribed CYP11B2*-positive solitary neoplasm } \\
\text { ( } \geq 10 \mathrm{~mm} \text { diameter) composed of clear or compact } \\
\text { eosinophilic cells or both cell types. }\end{array}$ \\
\hline Aldosterone-producing nodule & APN & $\begin{array}{l}\text { CYP11B2-positive lesion ( }<10 \mathrm{~mm} \text { diameter)** } \\
\text { morphologically visible with haematoxylin-eosin } \\
\text { staining. An APN often displays a gradient of CYP11B2 } \\
\text { immunostaining decreasing in intensity from the outer } \\
\text { to the inner part of the lesion. }\end{array}$ \\
\hline $\begin{array}{l}\text { Aldosterone-producing } \\
\text { micronodule (formally known as } \\
\text { aldosterone-producing cell cluster) }\end{array}$ & APM & $\begin{array}{l}\text { CYP11B2-positive lesion ( }<10 \mathrm{~mm} \text { diameter)** } \\
\text { composed of zona glomerulosa cells located beneath } \\
\text { adrenal capsule that do not differ in morphology from } \\
\text { adjacent adrenocortical cells by haematoxylin-eosin } \\
\text { staining. An APM often displays a gradient of CYP11B2 } \\
\text { immunostaining decreasing in intensity from the outer } \\
\text { to the inner part of the lesion. }\end{array}$ \\
\hline $\begin{array}{l}\text { Multiple aldosterone-producing } \\
\text { nodules or multiple aldosterone- } \\
\text { producing micronodules (formally } \\
\text { known as micronodular } \\
\text { hyperplasia) }\end{array}$ & $\begin{array}{l}\text { MAPN or } \\
\text { MAPM }\end{array}$ & $\begin{array}{l}\text { Multiple APN or multiple APM located beneath the } \\
\text { adrenal capsule with intermittent regions of normal zona } \\
\text { glomerulosa. MAPN and MAPM can coexist in the same } \\
\text { adrenal. }\end{array}$ \\
\hline $\begin{array}{l}\text { Aldosterone-producing diffuse } \\
\text { hyperplasia (APDH) }\end{array}$ & APDH & $\begin{array}{l}\text { Relatively broad and uninterrupted strip of zona } \\
\text { glomerulosa cells with more than half of these cells } \\
\text { displaying CYP11B2-positive immunostaining. }\end{array}$ \\
\hline
\end{tabular}

${ }^{*}$ CYP11B2= aldosterone synthase. CYP11B2 immunostaining must be performed using a well validated antibody (10)

**The histopathologic diagnosis of small lesions requires appropriate tissue sectioning to allow assessment of greatest diameter or dimension and to avoid distortion of lesion architecture 
Table 2. Clinical characteristics of patients stratified by histopathologic findings of classical and non-classical unilateral primary aldosteronism

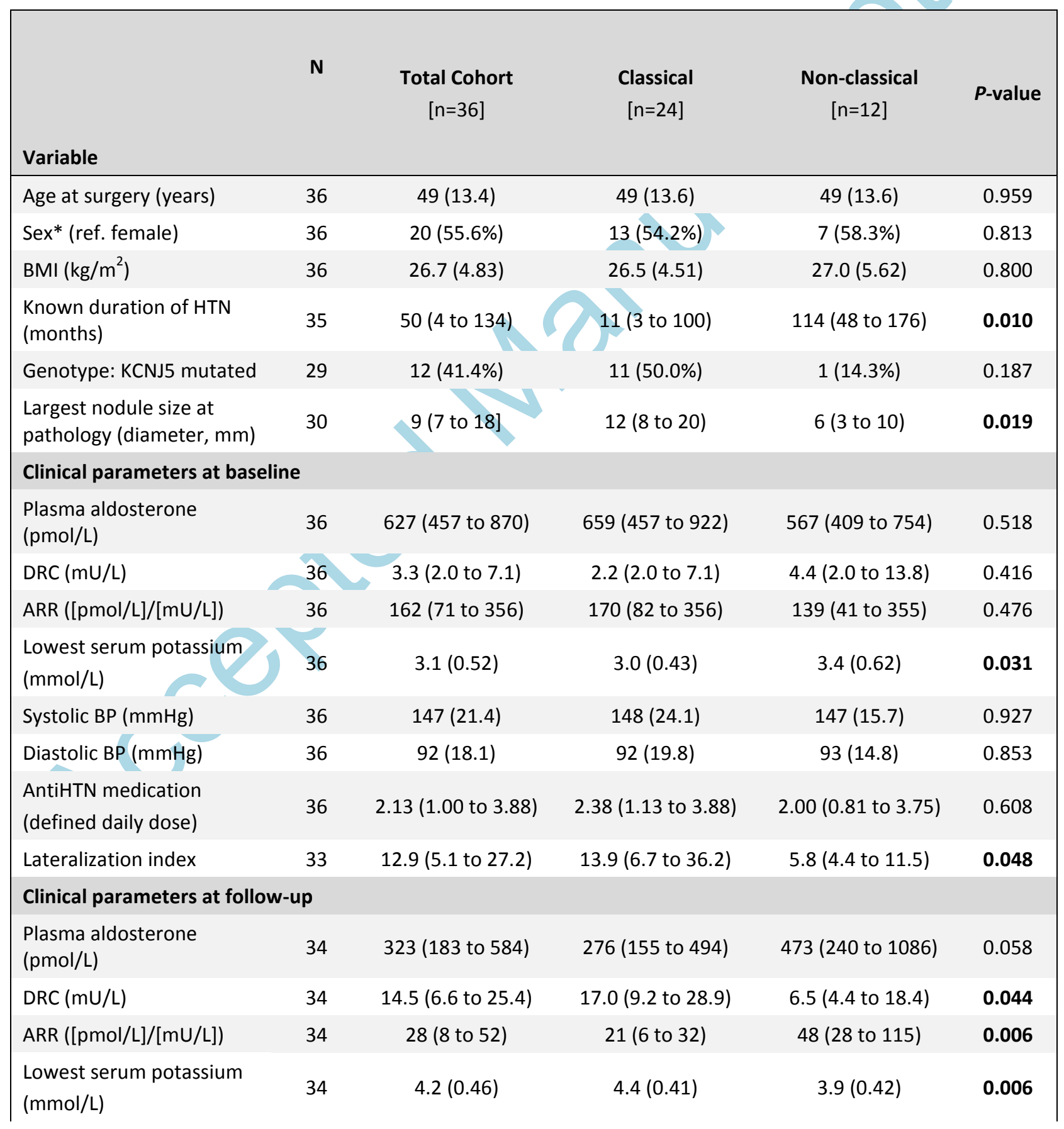




\begin{tabular}{|c|c|c|c|c|c|}
\hline Systolic BP (mmHg) & 34 & $136(16.2)$ & $134(17.2)$ & $140(14.0)$ & 0.338 \\
\hline Diastolic BP (mmHg) & 34 & $87(12.6)$ & $86(14.2)$ & $88(9.5)$ & 0.758 \\
\hline $\begin{array}{l}\text { AntiHTN medication } \\
\text { (defined daily dose) }\end{array}$ & 34 & $0.83(0.00$ to 2.13$)$ & 0.58 (0.00 to 2.53$)$ & 1.00 (0.50 to 1.88$)$ & 0.423 \\
\hline \multicolumn{6}{|l|}{ Clinical Outcome } \\
\hline Complete (n; \%) & & $4(11.8 \%)$ & $4(18.2 \%)$ & $0(0.0 \%)$ & \\
\hline Partial $(n ; \%)$ & 34 & $22(64.7 \%)$ & $13(59.1 \%)$ & $9(75.0 \%)$ & 0.286 \\
\hline Absent $(n ; \%)$ & & $8(23.5 \%)$ & $5(22.7 \%)$ & $3(25.0 \%)$ & \\
\hline \multicolumn{6}{|l|}{ Biochemical Outcome } \\
\hline Complete ( $\mathrm{n} ; \%)$ & & $22(64.8 \%)$ & $18(81.9 \%)$ & $4(33.3 \%)$ & $0.008 * *$ \\
\hline Partial (n; \%) & 34 & $6(17.6 \%)$ & $3(13.6 \%)$ & $3(25.0 \%)$ & $0.641^{* *}$ \\
\hline Absent $(n ; \%)$ & & 6 (17.6\%) & 1 (4.5\%) & 5 (41.7\%) & $0.014^{* *}$ \\
\hline
\end{tabular}

Unadjusted analysis of clinical parameters with data shown as the mean (SD), $\mathrm{n}(\%)$, or median (IQR). P values less than 0.05 were considered significant. Patient data from all cases of agreement between pathologists ( 36 of 37 resected adrenals with 25 cases of agreement between group 1 pathologists and 11 cases of agreement between group 2 pathologists (Figure 2). The remaining patient with disagreement between pathologists was excluded from the analysis.

Classical, indicates "classical" histopathology associated with unilateral PA of a solitary APA or APN; nonclassical, indicates "non-classical" histopathology of MAPM or MAPN (or MAPM and MAPN together) or APDH. Adrenal nodule diameter refers to the size of the largest nodule measured at pathology; lowest serum potassium refers to the lowest recorded serum potassium ion concentration and antihypertension medication is expressed as defined daily dose (DDD) which is the assumed average maintenance dose per day for a drug used for its main indication in adults (ATC/DDD Index 2010). The catheterization of the right adrenal gland was unsuccessful for three of 36 patients (lateralization indices could not be calculated). Follow-up measurements were unavailable for two of the 36 patients. Pairwise differences are shown for biochemical outcomes which displayed an overall group difference $(p=0.017)$. 
Genotype data was available for 29 of the 36 patients: a KCNJ5 mutation positive group $(n=12)$, and a KCNJ5 mutation negative group $(n=17)$. The latter group comprised those with no mutation detected $(\mathrm{n}=13), \operatorname{ATP} 1 A 1$ mutations $(\mathrm{n}=2)$, a CACNA1D mutation, and a PRKACA mutation. Clinical and biochemical outcomes after unilateral adrenalectomy were assessed in accordance with the PASO criteria at 6-12 months after surgery (24).

ARR, aldosterone-to-renin ratio; BMI, body mass index; BP, blood pressure; DRC direct renin concentration; HTN, hypertension; KCNJ5, potassium inwardly rectifying channel subfamily J member 5; ref., reference. ${ }^{*}$ The $p$-value is for sex in general; ${ }^{* *}$ overall $p$-value for biochemical outcomes=0.009. 
Figure 1

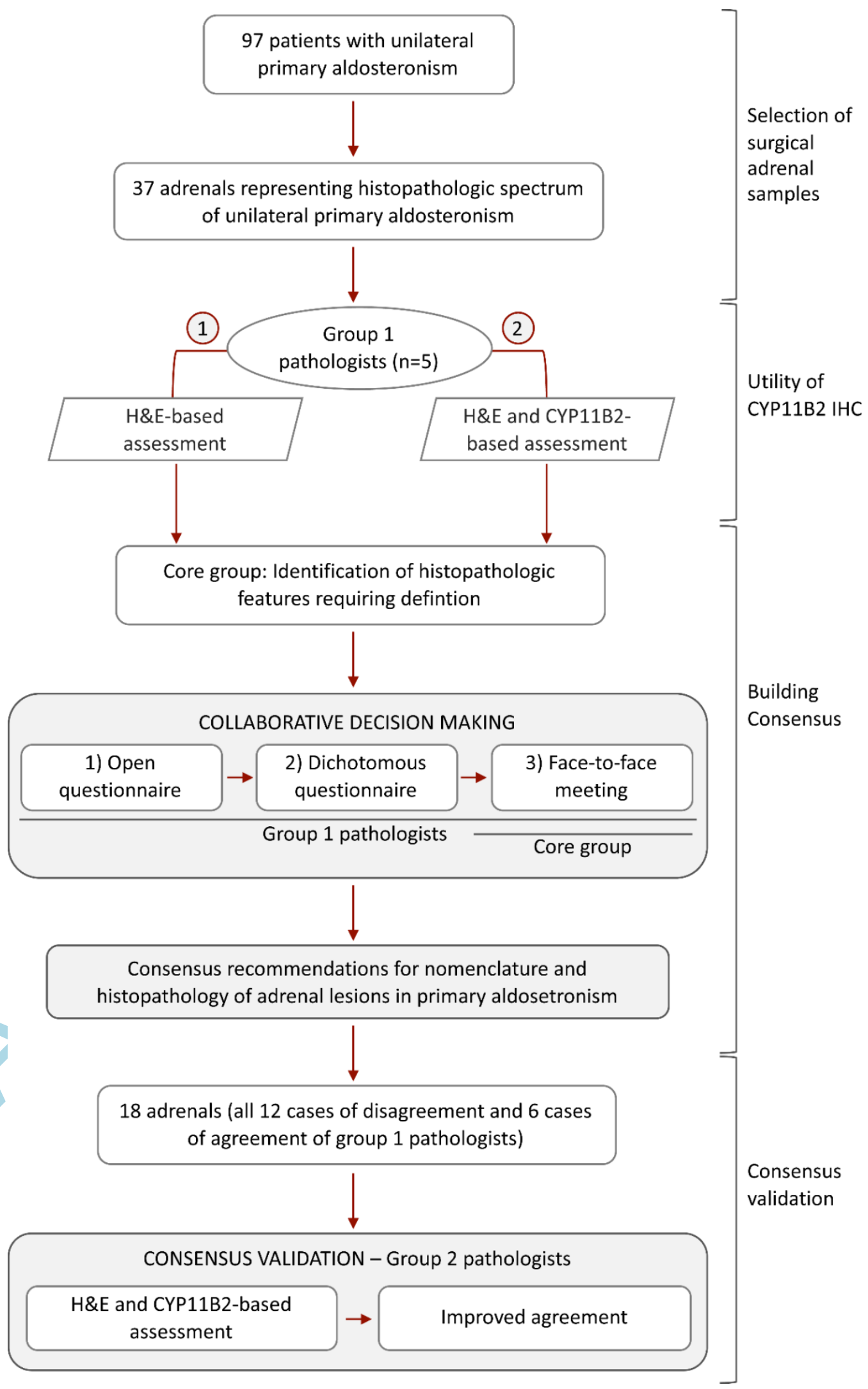


Figure 2

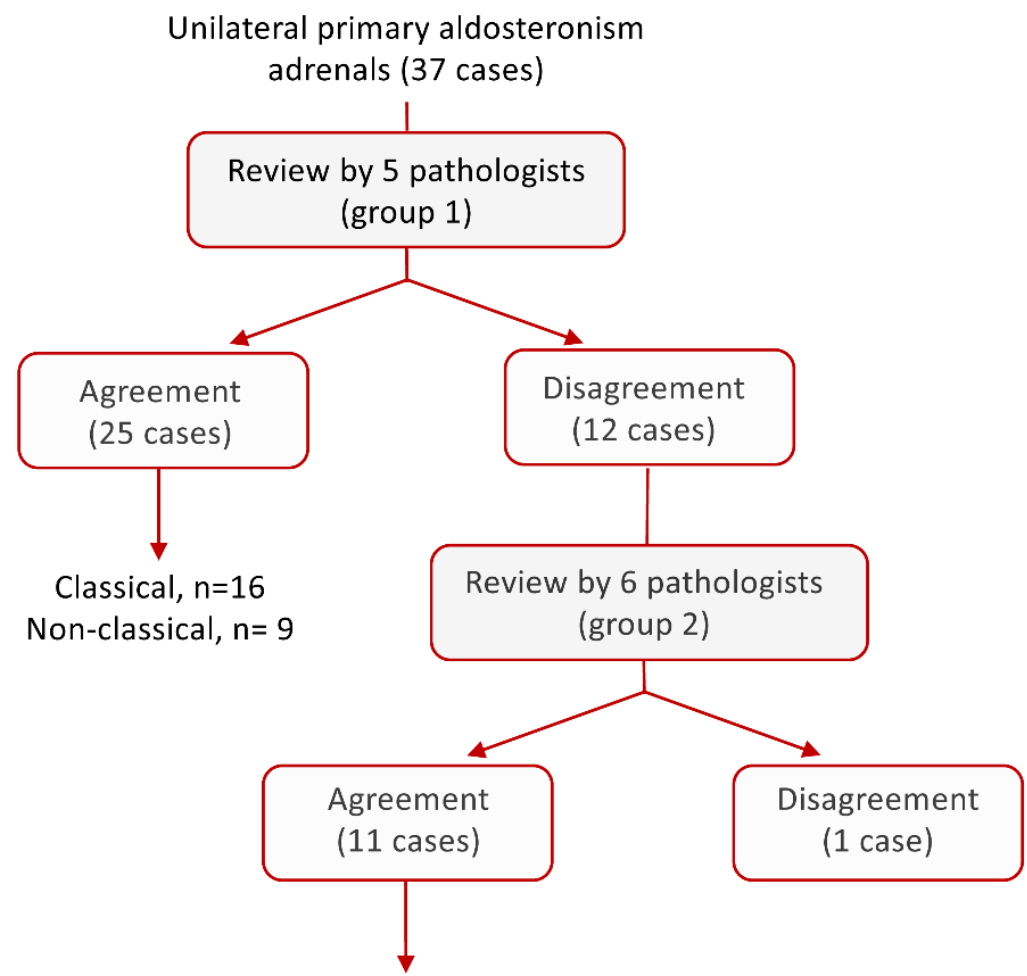

Classical, $\mathrm{n}=8$

Non-classical, $n=3$ 
Figure 3
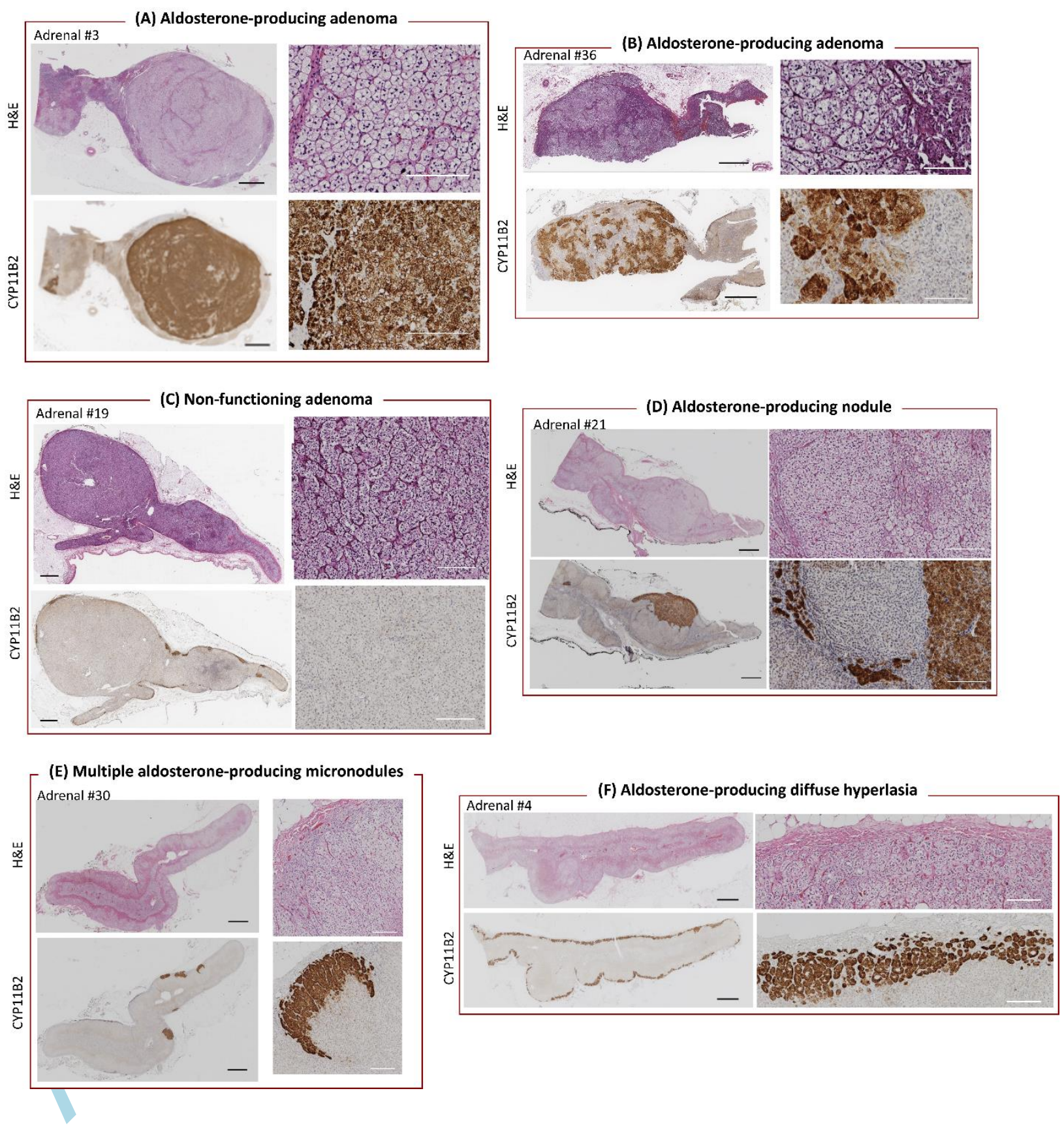